\title{
LA INCLUSIÓN LABORAL \\ DE LAS PERSONAS CON DISCAPACIDAD \\ DESDE LA ADMINISTRACIÓN PÚBLICA \\ RESPONSABLE EN LA COMUNIDAD \\ VALENCIANA
}

\section{The Labor Inclusion of People with Disability from the Responsible Public Administration in the Valencian Community}

Juli Antoni Aguado Hernández

Universitat de València. Facultat de Ciències Socials.

Departament de Sociologia i Antropologia Social

juli.aguado@uv.es

Susana Marín Traura

Universitat de València. Facultat de Filosofia i Ciències de l'Educació.

Departament de Teoria de l'Educació

Recepción: 6 de febrero de 2020

Aceptación definitiva: 11 de junio de 2020

REsumen: En la presente coyuntura socioeconómica, la posibilidad de inclusión a través del empleo de las personas con discapacidad es importante no solo por las posibilidades de autonomía económica, sino también por las oportunidades de socialización. Sobre esta base, y considerando que las empresas socialmente responsables asumen la inclusión de estas personas en sus plantillas como una posibilidad de crecimiento y de diferenciación, en el texto se analiza, de manera descriptiva, el papel que las administraciones públicas (AA. PP.) autonómicas, con competencias en la materia, pueden, deben y están haciendo para fomentar la responsabilidad social corporativa (RSC) y la gestión de la inclusión laboral de las personas con discapacidad. A partir de la discusión teórica y de la revisión de las políticas desarrolladas, se pretende dar respuesta a las siguientes cuestiones de fondo: ¿Cómo se está atendiendo la pluralidad en las empresas desde las administraciones públicas? ¿Son la RSC y la gestión de las personas con discapacidad modas pasajeras o por el contrario pueden resultar un instrumento válido de inclusión de estas personas dentro de los espacios de trabajo? Llegando a la conclusión de que esto solo será posible desde una RSC estratégica e integradora. 
Palabras Clave: discapacidad; empleo; inserción; inclusión; responsabilidad social corporativa.

Aвstract: At the present socio-economic juncture, the possibility of inclusion through the employment of people with disability is important not only because of the possibilities of economic autonomy, but also because of the opportunities for socialization. On this basis and considering that socially responsible companies take on the inclusion of these people in their workforce as a possibility of growth and differentiation, in the text is analyzed, in a descriptive way, the role that the autonomous Public Administrations (AA. PP.), with competences in the area, can, should and are doing to promote Corporate Social Responsibility (CSR) and management for the labor inclusion of people with disability. Based on the theoretical discussion and the review of the policies developed, is to provide replies to the following substantive questions: How is diversity being addressed in companies by public administrations? Are CSR and people with disability management fads or, on the contrary, can they be a valid instrument for the inclusion of these people within work spaces? Concluding that this will only be possible from a strategic and inclusive CSR.

KEY WORDs: disability; employment; insertion; inclusion; corporate social responsibility.

Todos los españoles tienen el deber de trabajar y el derecho al trabajo, a la libre elección de profesión u oficio, a la promoción a través del trabajo y a una remuneración suficiente para satisfacer sus necesidades y las de su familia.

Artículo 35 de la Constitución española (1978)

\section{Introducción}

$\mathrm{E}$

N LAS COMPlejas sociedades actuales las diferencias en las características personales, culturales y sociales están dejando de ser la excepción para convertirse en la norma. El reconocimiento pleno de derechos, como es el caso, por ejemplo, de las distintas minorías o de las personas con discapacidad, conduce a comunidades cada vez más diversas. Las empresas, que forman parte del tejido económico y social de estas sociedades, deben adaptarse a este nuevo entorno heterogéneo. Este es un importante reto, pero también una oportunidad evidente para el desarrollo sostenible de los negocios.

La "discapacidad" incluye a aproximadamente un $10 \%$ de la población ${ }^{1}$ y ha sido delimitada tanto por la Organización Mundial de la Salud (OMS) como por

1 Según la base de datos del Imserso, a 31 de diciembre de 2016, el 9,8 \% de la población española (4.563.749 personas de un total de 46.557.008) podría incluirse en este colectivo [http://www.ceapat.es/ InterPresent2/groups/imserso/documents/binario/bdepcd_2016.pdf], un porcentaje similar al de la población mundial (OIT, 2014; Raufflet et al., 2012). 
la Ley General de Derechos de las Personas con Discapacidad y su inclusión social $(\mathrm{LGDPD})^{2}$. No obstante, la literatura recoge también otro concepto que surge del propio colectivo: "diversidad funcional" (Palacios y Romañach, 2006). Esta propuesta intenta eliminar la concepción dualista entre personas discapacitadas $v s$. no discapacitadas (Patston, 2007). Las concibe como un continuo de funcionalidad, lo que disminuye la estigmatización y promueve una visión de valía e igualdad para cada persona que es diversa funcionalmente (Roosen, 2009). Desde esta perspectiva, se considera una diferencia con valor y no una carga, preservando su dignidad frente a la discriminación (Palacios y Romañach, 2006).

El presente texto expone la base teórica y las primeras indagaciones de un trabajo de investigación en curso desarrollado desde los Departamentos de Sociología y Antropología Social y de Teoría de la Educación de la Universitat de València. Teniendo en cuenta que las personas con discapacidad buscan la inserción laboral por las posibilidades de autodeterminación que les brinda, así como por las oportunidades de mejora en su calidad de vida social, profesional y personal y su bienestar general, se considera oportuno exponer el papel que las administraciones públicas autonómicas, con competencias en la materia, pueden, deben y están desempeñando con el fin de fomentar la responsabilidad social de las empresas y la inclusión laboral de las personas con discapacidad.

\subsection{Objetivo y metodología}

El marco teórico y el análisis de las políticas desarrolladas por la administración autonómica sobre la inserción laboral de las personas con discapacidad intenta responder a las siguientes cuestiones: ¿Cómo se está atendiendo la pluralidad en las empresas desde las administraciones públicas? ¿Son la responsabilidad social corporativa y la gestión de las personas con discapacidad simples modas pasajeras o por el contrario pueden resultar un instrumento válido de inclusión de estas personas en los espacios de trabajo?

Atendiendo a este objetivo, se desarrolla un análisis fundamentalmente descriptivo, mediante una metodología cualitativa basada en la técnica de recopilación documental (Ruiz e Ispzua, 1989; Valles, 1997), acudiendo a las diferentes fuentes del Servicio Valenciano de Empleo y Formación Autonómico (SERVEF, actualmente LABORA), con competencias en la materia. Este material incluye catálogos de servicios, memorias de actividades y planes estratégicos de los últimos diez años. Este estudio de las experiencias de atención a la gestión en materia de empleo mediante el análisis sistemático de estos materiales ha permitido adquirir conocimiento:

2 Según la OMS, las "deficiencias" son las limitaciones de las actividades y las restricciones en la participación. Es la interacción entre los individuos con un problema de salud y factores personales y ambientales (Aguilar y Yusta, 2017). Asimismo, en el art. 4.1, del RDL 1/2013, de 29 de noviembre, por el que se aprueba la LGDPD, estas personas se definen como aquellas que presentan deficiencias físicas, mentales, intelectuales o sensoriales, previsiblemente permanentes, que, al interactuar con diversas barreras, puedan impedir su participación plena y efectiva en la sociedad, en igualdad de condiciones con los demás. 
a) sobre quienes producen los documentos y su motivación -la propia administración (planes estratégicos)-; b) sobre el planteamiento de una administración enfocada al empleo y la formación -medidas concretas en discapacidad en las empresas y el resto de organizaciones, recursos destinados (catálogos de servicios, memorias de actividades)-; y c) sobre las condiciones sociales en las que se producen, su contexto, externo al propio contenido -necesidad de la incorporación de la responsabilidad social corporativa y la inclusión de las personas con discapacidad, retos planteados por y para la transformación del mercado laboral-.

\section{La discapacidad, la calidad de vida y la inclusión laboral}

El cambio desde una economía industrial hacia otra del conocimiento implica que, para ser competitivas, las empresas deben atraer, retener y desarrollar el talento de las personas creativas (Drucker, 1999); las tres T: tecnología, talento y tolerancia. Por otra parte, Batt y Valcour (2003) advierten que gestionar la inclusión de las personas con discapacidad como estrategia de negocio significa convertirla en uno de los cimientos de la responsabilidad de las organizaciones.

Entendiendo por tal los perfiles -visibles o no visibles- que diferencian a las personas que tienen un impacto en el comportamiento grupal (IEGD, 2019), la evolución del pensamiento corporativo sobre la misma es sintetizada por Thomas y Ely (1996) en los siguientes paradigmas: 1. Discriminación y Justicia; 2. Acceso y Legitimidad; 3. Aprendizaje y Eficacia. Esta última concepción implica una fuerza laboral plural en un mercado laboral heterogéneo, involucrando, asimismo, a las organizaciones en un continuo proceso de aprendizaje.

Por otro lado, en las últimas décadas ha aparecido uno nuevo: el de “inclusión”. Desde una perspectiva posestructuralista, según Díaz (2005), se trata de una actitud, un sistema de valores y creencias y no solo una acción o un conjunto de ellas. Implica reconocer unos ejercicios de poder, unos lenguajes y unas relaciones por deconstruir y reorientar. Exige ofrecer una propuesta de actuación que impida la transformación de las diferencias en marginación.

De igual forma, los aspectos fundamentales que conciernen a las personas con discapacidad están muy presentes actualmente en el impulso de fines comunes como los Objetivos de Desarrollo Sostenible 2030³.

En el caso de las personas con discapacidad, para su pleno desarrollo existen ciertas bases fundamentales entre las que destaca el derecho a trabajar en igualdad de condiciones con los demás (Forcada, 2015; Dalmau et al., 2015; Lukas et al., 2016), tal y como reconoce la Convención sobre los Derechos de las Personas con Discapacidad ${ }^{4}$.

\footnotetext{
3 En su introducción y en los ODS: 4, educación; 8, empleo; 10, reducción de las desigualdades; 11, inclusividad de las ciudades y accesibilidad del transporte, y 17, seguimiento de la Agenda.

4 Convención (Nueva York, 13/12/2006), ratificada por España (BOE n. ${ }^{\circ}$ 96, 21/04/2008), que incluye "el derecho a tener la oportunidad de ganarse la vida mediante un trabajo libremente elegido o aceptado en un mercado y un entorno laborales que sean abiertos, inclusivos y accesibles a las personas con discapacidad”.
} 
Desde el enfoque de capacidades de Sen (2002), los seres humanos no solo persiguen el bienestar, sino también “objetivos de agencia” más amplios, fijando metas al respecto y esforzándose por alcanzarlos. La calidad de vida tiene que ver con las cosas que pueden realmente "hacer" o "ser", los "funcionamientos" y sus "capacidades", las oportunidades para elegir y poder elegir llevar una u otra clase de vida.

En concreto, la OMS define la "calidad de vida" como un constructo complejo que recoge aspectos como la salud física de la persona, su estado psicológico, el nivel de independencia, las relaciones sociales o las creencias personales (Whoqol Group, 1995).

Asimismo, diferentes autores como Cummins (1997) y Felce y Perry (1995) proponen dominios y subdominios de la misma. Igualmente, según Claes et al. (2012), para alcanzar niveles saludables de calidad de vida las personas con discapacidad deben poder disfrutar de independencia personal, participación social y bienestar general.

Para Schalock y Verdugo (2007) esta consiste en un estado de bienestar personal que es multidimensional: con propiedades universales y conectadas a la cultura; con componentes objetivos y subjetivos, e influido tanto por características personales como por factores ambientales. A partir de este modelo, los autores identifican ocho dimensiones esenciales de calidad de vida importantes para todas las personas: bienestar emocional, bienestar físico, bienestar material, relaciones interpersonales, desarrollo personal, autodeterminación, inclusión social y derechos (Lukas et al., 2016; Morán et al., 2019).

Por otra parte, la “inserción laboral” puede entenderse, en general, como el proceso o transición de incluir a las personas con la edad correspondiente en el sistema ordinario de trabajo (Sánchez et al., 2016). Siguiendo los modelos de Schalock y Verdugo (2007) y de Claes et al. (2012), la generación de oportunidades laborales permite a las personas con discapacidad mejorar su calidad de vida social, profesional y personal y, por lo tanto, su autodeterminación, su bienestar general y su constitución como ciudadanía con plenos derechos. Por consiguiente, también enriquece los resultados personales, la promoción del beneficio que cada persona puede aportar a la comunidad desde el ejercicio de un rol social valorado (Tamarit, 2015; Lukas et al., 2016; González, 2019).

En este sentido, la participación social y la inclusión laboral son fundamentales para el desarrollo de las personas con discapacidad, para su inclusión en la comunidad y el desarrollo de una vida independiente (Thomas y Ely, 1996; Izuzquiza y Rodríguez, 2016; Vived y Delgado, 2016); en definitiva, para conseguir una mayor calidad de vida.

Sin embargo, pese a su impacto en todas estas dimensiones de la calidad de vida, el acceso al mundo laboral sigue implicando un importante escollo para personas con discapacidad (Mercado et al., 2016; Gónzalez, 2019). En efecto, si hace una década ${ }^{5}$ solo trabajaban un $24 \%$ de estas personas mayores de 16 años frente al $65,3 \%$ de la población en general (Castillo y Suso, 2012, p. 5), la situación no ha variado mucho en la actualidad. El análisis de Aguilar y Yusta (2017) sobre la tasa

5 Datos de la EPA del INE de 2008. 
de actividad del año 2015 muestra una importante diferencia entre las tasas de las personas con y sin discapacidad (en las mujeres esta diferencia es de un $40 \%$ y en los hombres de un 50 \%). Asimismo, según el SERVEF SER $^{6}$ (2016, p. 20), de 2009 a 2014 el contingente de personas activas de este colectivo en la Comunitat Valenciana asciende de 32.400 a 60.600 , y su número de personas desempleadas registradas aumenta de 9.238 en 2009 a 17.448 en 2015. Esta pauta se repite en los/as jóvenes (Dalmau et al., 2015; Fund. Adecco, 2018).

Esta diferencia podría atribuirse, entre otras cosas, a las actitudes de los empleadores (Raufflet et al., 2012); a la sobreprotección familiar; al desconocimiento y la falta de recursos para la búsqueda de empleo (Fund. Adecco, 2018); al menor número de puestos de trabajo existentes, o al efecto renta que provoca la disminución de la participación en el mercado de trabajo al tener reconocida una pensión (Rubio, 2006).

Esta realidad de "exclusión” (Mercado et al., 2016) es reconocida por diferentes organismos internacionales ${ }^{7}$ y se refleja en fuentes estadísticas como el Eurobarómetro ${ }^{8}$.

Precisamente por todo ello, para aportar propuestas de actuación que impidan la transformación de las diferencias en marginación (Díaz, 2005), es necesario diseñar mecanismos y herramientas para atender las especiales necesidades y facilitar el acceso al empleo, la participación social y la vida autónoma de las personas con discapacidad (Mercado et al., 2016; Vived y Delgado, 2016).

\section{La responsabilidad social corporativa y la gestión de la diversidad}

En la discusión sobre estos temas puede entreverse la confusión entre la responsabilidad social corporativa y la gestión de la diversidad, dos marcos diferentes, pero con obvias conexiones entre sí. Por una parte, la "gestión de la diversidad" puede definirse como una "estrategia corporativa destinada a la creación de un soporte incluyente para los perfiles diversos de las personas, que optimice la eficacia del proceso empresarial” (Castillo y Suso, 2012, p. 36). También, como un compromiso corporativo, una estrategia integral basada en la creación de una población laboral de perfiles diversos en las organizaciones. Este compromiso de inclusión delimita la capacidad de una empresa con el fin de integrar a su plantilla para avanzar hacia objetivos comunes y compartidos (IEGD, 2019).

Por otra parte, una de las definiciones más consensuadas (Carneiro, 2004; Fund. Alternativas, 2009; Chaves y Monzón, 2018) de la responsabilidad social corporativa es la del Libro Verde de la Comisión Europea (CCE, 2001), que informa sobre su carácter voluntario y que entiende por tal:

6 Datos de la operación estadística del INE "El empleo en las personas con discapacidad": https:// www.ine.es/dyngs/INEbase/es/operacion.htm?c=Estadistica_C\&cid=1254736055502\&menu=ultiDatos \&idp $=1254735976595$.

Convenio 111 Discriminación en materia de Empleo y Ocupación de la ONU (1958), Tratado de Ámsterdam (1997), el Libro verde de la Comisión Europea sobre la responsabilidad social (CCE, 2001).

8 Siendo la discapacidad (con un $53 \%$, en UE27 y un $56 \%$ en España) la tercera causa por la que se han sentido discriminadas más frecuentemente (Castillo y Suso, 2012, pp. 30-31). 
la integración voluntaria, por parte de las empresas, de las preocupaciones sociales y medioambientales en sus operaciones comerciales y en sus relaciones con todos sus interlocutores, yendo más allá de su cumplimiento [de las obligaciones jurídicas] invirtiendo "más" en el capital humano, el entorno y las relaciones con los interlocutores (p. 7).

Posteriormente, la Comisión presenta una nueva definición de la misma: "La responsabilidad de las empresas por su impacto en la sociedad” (CE, 2011, p. 7), incorporando el compromiso empresarial por su "incidencia” sobre las comunidades.

Más concretamente, estamos hablando de un proceso de gestión, de inversión y de comportamiento de las organizaciones que incluyan dispositivos de evaluación del desempeño y de incentivación que primen la dimensión social (Chaves y Monzón, 2018) en su relación con los stakebolders internos y externos. Lo que conlleva una nueva forma de administrar sus externalidades en los ámbitos económico, social y medioambiental y de respeto a los Derechos Humanos (Melle, 2014; Fund. Cepaim, 2016).

Las ventajas de implantar políticas responsables en las empresas son considerables para la reactivación económica por sus aportaciones en el progreso del conocimiento, la relación con los interlocutores, la reputación, el control de riesgos, la mejora de ventajas competitivas como factor de diferenciación, etc. (Fund. Alternativas, 2009). Pero también para asumir la pluralidad de sus plantillas no como un hándicap, sino como una posibilidad de crecimiento y de diferenciación frente a otro tipo de competencia más anquilosada y con parámetros más obsoletos (Drucker, 1999; Batt y Valcour, 2003).

\section{La responsabilidad social corporativa en las administraciones públicas}

Lo social está intrínsecamente unido a lo público, sobre todo si hablamos de cualquiera de las administraciones estatales, por lo que las actuaciones que van en contra del bien común no pueden estimarse como responsables socialmente (González y Naranjo, 2015).

En este sentido, la responsabilidad social ha evolucionado hacia una "integración social corporativa” de carácter copernicano (Porter y Kramer, 2006, p. 92), en la que las empresas son parte, pero no su centro, pues las administraciones y la sociedad también son responsables. Se ha convertido, así, en un modelo de gestión transversal a lo público, privado tradicional y de tercer sector (Chaves y Monzón, 2018), pasando a formar parte de las prácticas del sector público (Morros y Vidal, 2005; Melle, 2014; Canyelles, 2011).

\subsection{Papel económico y responsabilidad de las administraciones públicas}

Más allá de hacer las cosas bien desde un punto de vista normativo y de eficacia, se hace referencia a la forma en que se implementan las políticas, de forma sostenible, aportando valores como transparencia, información comprensible y adaptada, mayor 
atención a la ciudadanía, de manera ética, en diálogo con los grupos de interés, etc.; con independencia de si existe o no una obligación legal para ello (Canyelles, 2011; Melle, 2014).

Las administraciones públicas no son ajenas a la realidad de un mundo globalizado ni a las crisis económicas como las vividas hasta ahora, sino que interactúan en un entorno social, económico y ambiental. Por ello, deben ser cada vez más eficientes e innovar en sus servicios, en las respuestas a los retos, demandas e inquietudes que tienen planteados (Canyelles, 2011; EERSE, 2014) como es el caso de la inclusión de los colectivos más vulnerables o con dificultades de acceso a derechos tales como el del trabajo.

Las administraciones públicas tampoco son extrañas a los requerimientos de organizaciones sociales y políticas ni a los usuarios y las usuarias de servicios del siglo $\mathrm{XXI}$, que demandan su reconocimiento como sujetos de derechos y una gestión ética, transparente y sostenible (Canyelles, 2011; González y Naranjo, 2015). Mejora de los servicios que, en el caso de los enfocados hacia la calidad de vida de las personas con discapacidad (Schalock y Verdugo, 2007; Tamarit, 2015), juega un papel fundamental en su satisfacción y bienestar (Gracia et al., 2017).

Por otra parte, las administraciones gubernamentales son un agente económico de una importancia excepcional, tanto en el terreno del empleo como en el de gestión de sus propios fondos o como inversor en ámbitos de interés (CD, 2006). Inciden de forma significativa en las diferentes instituciones y organizaciones, incluyendo sus stakebolders (González y Naranjo, 2015). Por lo tanto, sus actuaciones tienen unos importantes impactos económicos, sociales, ambientales, laborales y de reputación (Melle, 2014).

En esta línea, la Estrategia Española de Responsabilidad Social 2014-2020 (EERSE, 2014) no concreta la responsabilidad social en las administraciones públicas, pero presenta un marco conceptual para estas organizaciones que:

... prestan servicios a los ciudadanos y, por tanto, deben ser cada vez más eficientes en su actividad puesto que interactúan en un entorno social y ambiental que deben respetar y mejorar, al tiempo que deben velar por el desarrollo profesional y el bienestar laboral de los empleados públicos.

Por todo ello, las Administraciones, en tanto que son organizaciones, deben aplicarse a sí mismas los mismos criterios que inspiran el concepto de responsabilidad social y hacerlo, además, con un carácter de ejemplaridad (p. 22).

\section{Actuaciones responsables de las administraciones públicas}

La Comisión Europea (CE, 2014), entre los retos de España en materia de empleo, contempla como prioritarias las acciones para facilitar la integración en el mercado laboral de las personas desfavorecidas (incluidas las de etnia gitana, personas inmigrantes y las personas con discapacidad).

Las directrices europeas dejan clara constancia de la responsabilidad gubernamental acerca de la necesidad de mejora y eficacia de los servicios públicos de empleo 
dirigidos a las personas desempleadas, especialmente a los colectivos en situación de vulnerabilidad (p. e., personas con discapacidad) mediante políticas activas. Pero esta gestión de las personas en el ámbito público y privado debe hacerse en torno a las competencias $^{9}$ y las administraciones españolas necesitan incorporar este enfoque.

Entre las medidas concretas que pueden tomar los poderes públicos destacan dos amplias vías de actuación. Por un lado, prestando atención a su propio funcionamiento y modelo, por medio de un claro compromiso con la responsabilidad social como herramienta estratégica en la gestión, más allá de sus obligaciones. Pero, por otro lado, deben generar un entorno favorable para las estrategias responsables mediante actuaciones reguladoras y de fomento, estímulo, difusión y apoyo en el ámbito de las empresas y del resto de organizaciones públicas y privadas (Raufflet et al., 2012; EERSE, 2014; Melle, 2014).

\subsection{El compromiso propio con la responsabilidad social por parte de las administraciones públicas}

Según la OIT (2014), desarrollar el principio de la igualdad de oportunidades y de trato supone, en el caso estudiado, adaptar el entorno y tomar medidas especiales mediante acciones positivas para evitar la discriminación. Estas actuaciones conllevan un trato preferencial para quienes tienen necesidades particulares en aras de su mayor inclusión (Carneiro, 2004; Raufflet et al., 2012).

En cuanto al compromiso propio con la responsabilidad social por parte de las administraciones públicas, como se ha indicado, estas están integrando en mayor medida estos principios en sus propios sistemas de gestión, atendiendo a las demandas sociales y a las recomendaciones de la Comisión de la UE (CCE, 2001, 2011) y la Subcomisión parlamentaria española (CD, 2006), acompasándose así con la lógica evolutiva hacia sistemas de buen gobierno corporativo (Canyelles, 2011; Melle, 2014).

En el caso del ámbito "interno" de las organizaciones, estamos hablando de las nuevas tendencias en la gestión de los recursos humanos desde una perspectiva responsable. Los trabajadores son el stakebolder más próximo al núcleo de la organización (Carneiro, 2004; Raufflet et al., 2012; Castillo y Suso, 2012) y son el área en la que se plantean con frecuencia problemas éticos (Ruiz Otero et al., 2012). De hecho, una organización comprometida con su plantilla es más sostenible y rentable (Raufflet et al., 2012).

En este ámbito interno, en el marco del compromiso ético, del respeto hacia la pluralidad e igualdad de oportunidades, deben apostar por planes de inclusión social de colectivos vulnerables, favoreciendo su contratación o limitando la subcontratación (Melle, 2014; Castillo y Suso, 2012). En el caso de estudio, según la LGDPD, las empresas que empleen a un número de 50 o más personas trabajadoras vendrán obligadas a que de ellas al menos el $2 \%$ lo sean con discapacidad.

9 Por ejemplo, según el Foro de Vida Independiente y Diversidad: http://forovidaindependiente.org/. 
En cuanto a la contratación pública, la legislación comunitaria ${ }^{10}$ incluye cláusulas de tipo social y ambiental como condiciones especiales de ejecución del contrato o como criterios para valorar las ofertas, entre otras materias, mediante la contratación de personas con dificultades de inserción (ORSC, 2011; EERS, 2014; Melle, 2014).

Asimismo, la Ley de Contratos del Sector Público ${ }^{11}$ (LCSP) plasma los mecanismos que permiten utilizar la capacidad económica de las administraciones para la consecución de objetivos de interés social y general, como la inserción de personas con discapacidad, al igual que la ley valenciana para el fomento de la responsabilidad social $^{12}$.

Estas estipulaciones pueden ser elementos de valoración en distintas fases del proceso de contratación pública: como requisito previo en los criterios de admisión y selección de las empresas (p. e., puntuando el compromiso de contratar personas trabajadoras con dificultades de inserción laboral), como elemento de valoración en la adjudicación (p. e., exigiendo experiencia o solvencia técnica en la contratación de colectivos vulnerables) y como una obligación consustancial al contrato en sus cláusulas de ejecución (p. e., contratación de cierto número de personas en situación de riesgo social) (ORSC, 2011; EERS, 2014).

Junto con estas cláusulas, la LCSP destaca la figura jurídica de la "reserva de contratación”. Así, en la Disposición Adicional 4. ${ }^{\text {a establece: }}$

porcentajes mínimos de reserva del derecho a participar en los procedimientos de adjudicación de determinados contratos o de determinados lotes de los mismos a Centros Especiales de Empleo de iniciativa social y a empresas de inserción [...] o un porcentaje mínimo de reserva de la ejecución de estos contratos en el marco de programas de empleo protegido.

\subsection{La Administración pública como impulsora y dinamizadora de la responsabilidad social}

La inclusión de las personas con discapacidad puede convertirse en una ventaja competitiva sostenida. El informe de Cox y Blake (1991), la Comisión Europea (The Conference Board, 2006; IEGD, 2019) o diferentes autores (Castillo y Suso, 2012) coinciden en que las empresas que aplican políticas de respeto por la pluralidad pueden generar beneficios como:

- Reducción de costes por menores niveles de rotación y absentismo ${ }^{13}$.

- Mejora cualitativa y/o cualitativa en la atracción y la retención del talento.

- Equipos plurales que afrontan con mayores ventajas la solución de problemas, aumento de la creatividad y de la innovación.

10 Directivas 2004/18/CE del Parlamento Europeo y del Consejo, de 31/03/2004, sobre Coordinación de los Procedimientos de Adjudicación de los Contratos Públicos de Obras, Suministros y Servicios; y 2014/24/UE del Parlamento Europeo y del Consejo de 26/02/2014 sobre contratación pública.

11 Ley $9 / 2017$, de 8 de noviembre, de Contratos del Sector Público.

12 Ley 18/2018, de 13 de julio, de la Generalitat, para el fomento de la responsabilidad social.

13 Sobre el menor absentismo en la plantilla con discapacidad véase: Fund. Adecco (2015). 
- Potenciación del cambio cultural y fomento del intercambio de conocimiento.

- Mejora del clima laboral.

- Organizaciones favorecedoras de la flexibilidad interna.

- Refuerzo de la imagen de marca y de la reputación corporativa.

- Incremento de la productividad y de la eficiencia y la eficacia empresarial.

- Aumento de las oportunidades de mercado y acceso a nuevos mercados.

- Mejoras en las respuestas de los clientes y en su fidelización.

- Reducción de costes legales.

Estas actuaciones pueden incluirse en la responsabilidad social "estratégica". Según Porter y Kramer (2006), esta es una herramienta para generar valor en el largo plazo y obtener ventajas competitivas duraderas para las empresas como una buena reputación, una posición diferenciada en el mercado, innovar sus procesos y productos reduciendo costes o reclutar personal cualificado. Es una nueva forma de concebir el negocio y sus estrategias que considera que su desarrollo económico debe ir unido a mejoras para la sociedad, siendo inseparable de las relaciones de confianza con los stakeholders.

Sobre esta base, en el papel de las administraciones públicas como impulsoras, dinamizadoras o catalizadoras para generar un entorno favorable y el fortalecimiento de la responsabilidad pueden distinguirse cuatro categorías básicas de actuaciones (Fox et al., 2002; CD, 2006): reguladora, facilitadora o de apoyo, colaboradora y promotora.

En el ámbito regulador, se contempla el marco normativo que define los estándares mínimos de la actuación empresarial y de la aplicación de la responsabilidad social en las organizaciones. En concreto, pueden destacarse, en primer lugar, la aplicación de la LGDPD, por la que las empresas de más de 50 personas trabajadoras están obligadas a que entre ellas al menos el $2 \%$ lo sean con discapacidad.

En segundo lugar, las bonificaciones o reducciones y las subvenciones o ayudas públicas para el fomento del empleo de determinados colectivos, destacando en el ámbito de la discapacidad las bonificaciones de las cuotas a la Seguridad Social de los contratos temporales de fomento de empleo, indefinidos y para la formación y el aprendizaje (SEPE, 2019). Sin olvidar las ayudas para el fomento del empleo autónomo para personas con discapacidad, tal como se describen en el próximo apartado ${ }^{14}$.

En tanto que facilitadoras, las administraciones públicas pueden estimular la implicación de actores clave en la responsabilidad social, mediante medidas de fomento o de impulso de la misma (incentivos, formación, comunicación, difusión de información, divulgación de buenas prácticas, etc.). Entre otras fórmulas, pueden fomentarse actividades de acción social por las empresas en diferentes áreas, relacionadas o no con el sector de actividad donde operan, como la atención social, la salud, la educación y el empleo (Carneiro, 2004; Morrós y Vidal, 2005). También, favoreciendo la inserción laboral mediante acuerdos de colaboración con empresas de tecnología y las TIC (Forcada, 2015).

En su vertiente colaboradora, pueden actuar mediante alianzas y la puesta en común de capacidades y logros complementarios a los de las iniciativas privadas y de la sociedad civil, entre estas organizaciones o entre ellas y las propias administraciones,

14 http://www.labora.gva.es/es/empreses/busque-ajudes-subvencions/ajudes-foment-de-l-ocupacio. 
que pueden actuar como participantes, coordinadoras o facilitadoras del partenariado. En este sentido, pueden favorecer las alianzas entre negocios, asociaciones y ONG o reforzar sus propias relaciones con las entidades del tercer sector o de iniciativa social dedicadas a colectivos vulnerables, pues, a través de estas últimas, muchas empresas ya buscan la generación de valor social mediante proyectos de desarrollo social y local (Raufflet et al., 2012).

En su papel de promotoras de las iniciativas que ponen en práctica la responsabilidad social corporativa, pueden actuar mediante fórmulas de reconocimiento directo de los esfuerzos, de formación de directivos, de financiación de consultoras para asesoría a pymes y el fomento de las metodologías para su implantación. Algunas empresas ya establecen políticas para personas capacitadas para los puestos, pero con especiales dificultades de acceso al empleo (Morrós y Vidal, 2005; Ruiz Otero et al., 2012), comprobando que los costes de adaptación no son elevados (Raufflet et al., 2012) ) $^{15}$.

También, mediante el apoyo a los Centros Especiales de Empleo (CEE) o empresas de inserción ${ }^{16}$ con la finalidad de crear oportunidades laborales para la población con discapacidad que permitan desarrollar mejores condiciones de calidad de vida, tanto en el propio puesto de trabajo como en su vida en general (Lukas et al., 2016), al amparo de las orientaciones del artículo 35 de la LGDPD.

Igualmente, considerando las ventajas del empleo en empresas ordinarias (Vived y Delgado, 2016), mediante el fomento del empleo con apoyo, regulado por el RD 870/2007, de 2 de julio. Este modelo de inclusión contribuye al acceso y la consolidación del empleo de personas con especiales necesidades en entornos competitivos, asegurando que los/as trabajadores/as y las empresas tengan un apoyo en la figura del mediador o preparador laboral a lo largo de todo el proceso de inserción (Dalmau et al., 2015; Izuzquiza y Rodríguez, 2016; Mercado et al., 2016).

\subsection{Los servicios públicos de empleo y otras iniciativas de mejora de la empleabilidad de la Generalitat Valenciana}

La importancia de los servicios para el empleo destinados a las personas con discapacidad y su relación con el concepto de calidad de vida están vinculadas directamente con las políticas públicas sociales (Schalock y Verdugo, 2007; Lukas et al., 2016; Morán et al., 2019) y, por consiguiente, con las políticas de ocupación.

En cuanto a las administraciones como servicio público de empleo, en el ámbito autonómico valenciano, del análisis del Catálogo de servicios del SERVEF (2010) y de diferentes documentos de trabajo del organismo autónomo ${ }^{17}$ puede destacarse que la inclusión de la discapacidad en el mismo aparece en los siguientes apartados, asumiendo así que la capacitación es una herramienta esencial para la integración laboral:

15 Pueden consultarse buenas prácticas en contratación de personas con discapacidad e inclusión en: BA (2012a, 2012b); así como en: https://fundacionadecco.org/azimut/category/buenas-practicas/.

16 http://www.labora.gva.es/es/centres-especials-d-ocupacio; http://www.labora.gva.es/es/entitats/ busque-ajudes-subvencions/empreses-d-insercio.

17 “130_A05_Información formación” y “210_A11_Autoempleo”. 


\section{TABLA 1. La discapacidad en el Catálogo de servicios del SERVEF}

\begin{tabular}{|l|l|}
\hline \multicolumn{1}{|c|}{ Programa } & \multicolumn{1}{c|}{ Destinatarios } \\
\hline $\begin{array}{l}\text { Formación profesional para el } \\
\text { empleo (FPO). }\end{array}$ & $\begin{array}{l}\text { Colectivos prioritarios a efectos de su participación, } \\
\text { entre otros, personas con discapacidad. }\end{array}$ \\
\hline & $\begin{array}{l}\text { El alumnado con discapacidad tendrá derecho a una } \\
\text { ayuda por día asistido. }\end{array}$ \\
\hline $\begin{array}{l}\text { Talleres de formación e inserción } \\
\text { laboral (TFIL). }\end{array}$ & Tendrán preferencia las personas con discapacidad. \\
\hline $\begin{array}{l}\text { Formación profesional para el } \\
\text { empleo dirigido prioritariamente a a } \\
\text { trabajadores ocupados. }\end{array}$ & $\begin{array}{l}\text { Prioridad para participar como alumnado, entre otros, } \\
\text { personas discapacitadas en grado igual o superior al } \\
33 \% .\end{array}$ \\
\hline
\end{tabular}

FUENTE: SERVEF (2010). Elaboración propia.

TABLA 2. La discapacidad en el Plan Estratégico del SERVEF

\begin{tabular}{|c|l|l|}
\hline Indicador & \multicolumn{1}{|c|}{ Descripción } & \multicolumn{1}{|c|}{ Variables } \\
\hline I.1.4.2 & $\begin{array}{l}\text { Relación porcentual entre el número de } \\
\text { contratos fijos registrados por personas } \\
\text { con discapacidad y el total de contratos } \\
\text { registrados por personas con discapacidad } \\
\text { durante el periodo de referencia. }\end{array}$ & $\begin{array}{l}\text { V.o14. Número de contratos } \\
\text { fijos registrados por } \\
\text { personas con discapacidad. } \\
\text { V.o15. Número total de } \\
\text { contratos registrados por } \\
\text { personas con discapacidad. }\end{array}$ \\
\hline I.1.4.4 & $\begin{array}{l}\text { Relación porcentual entre el incremento } \\
\text { relativo de contratos registrados para el } \\
\text { colectivo de personas con discapacidad y } \\
\text { el incremento relativo total de contratos } \\
\text { registrados durante el periodo de referencia. }\end{array}$ & $\begin{array}{l}\text { V.o15. Número total de } \\
\text { contratos registrados por } \\
\text { personas con discapacidad. }\end{array}$ \\
\hline I.1.4.6 & $\begin{array}{l}\text { Indicador de síntesis que aglutine con } \\
\text { ponderaciones predeterminadas los } \\
\text { indicadores anteriores, con el objeto de } \\
\text { resumirla información en este apartado. }\end{array}$ & $\begin{array}{l}\text { V.015. Número total de } \\
\text { contratos registrados por } \\
\text { personas con discapacidad. }\end{array}$ \\
\hline I.1.4.7 & $\begin{array}{l}\text { Relación porcentual entre el gasto } \\
\text { dedicado a financiar o mantener el empleo } \\
\text { de colectivos prioritarios (personas con } \\
\text { discapacidad, mujeres, riesgo de exclusión } \\
\text { social)y el total de gasto destinado a } \\
\text { financiaro subvencionar el empleo. }\end{array}$ & $\begin{array}{l}\text { V.o18. Gasto dedicado } \\
\text { a financiar o mantener } \\
\text { el empleo de colectivos } \\
\text { prioritarios (personas con } \\
\text { discapacidad, mujeres, riesgo } \\
\text { de exclusión social). }\end{array}$ \\
\hline I.1.4.8 & $\begin{array}{l}\text { Relación porcentual entre el número } \\
\text { de personas pertenecientes a colectivos } \\
\text { prioritarios (personas con discapacidad, } \\
\text { mujeres, riesgo de exclusión social) con } \\
\text { contratos subvencionados y el total de } \\
\text { personas con contratos subvencionados. }\end{array}$ & $\begin{array}{l}\text { V.o53. Número de personas } \\
\text { pertenecientes a colectivos } \\
\text { prioritarios (personas con } \\
\text { discapacidad, mujeres, riesgo } \\
\text { de exclusión social) con } \\
\text { contratos subvencionados. }\end{array}$ \\
\hline
\end{tabular}

Fuente: SERVEF (2016). Elaboración propia. 
Por otra parte, la discapacidad aparece en el Plan Estratégico del SERVEF 20162022 (SERVEF, 2016) en los siguientes indicadores del Eje 1. Servicios, cuyo Objetivo Estratégico 1.4 es Fomentar y sostener la contratación de calidad. Remover obstáculos $y$ fomentar y promover la igualdad de oportunidades en el acceso al empleo.

Atendiendo a los recursos existentes, en el Plan estratégico de subvenciones 20172019 del actual LABORA (SERVEF, 2017) constan las siguientes líneas presupuestarias.

\begin{tabular}{|c|c|c|c|c|c|c|c|c|c|}
\hline \multicolumn{10}{|c|}{$\begin{array}{l}\text { TABLA 3. Programa presupuestario: } 322.51 \text { Fomento de Empleo (Importes anuales, } \\
\text { vinculación con los objetivos operativos y fuentes de financiación) }\end{array}$} \\
\hline $\begin{array}{l}\text { Código } \\
\text { Línea }\end{array}$ & Cap. & Descripción & Obj. & Solicitantes & $\begin{array}{c}\text { Colectivo } \\
\text { destinatario } \\
\text { Participantes }\end{array}$ & $\begin{array}{c}\text { Importe } \\
\text { proyecto } \\
2017\end{array}$ & $\begin{array}{l}\text { Importe } \\
\text { proyecto } \\
2018 \\
\end{array}$ & \begin{tabular}{|c|} 
Importe \\
proyecto \\
2019
\end{tabular} & Fin. \\
\hline S2240000 & 4 & $\begin{array}{l}\text { Empleo de personas } \\
\text { con diversidad } \\
\text { funcional, enfermos } \\
\text { mentales e inclusión } \\
\text { social. }\end{array}$ & A. 3 & $\begin{array}{l}\text { Centros Especiales de Empleo, } \\
\text { Entidades Locales e instituciones } \\
\text { sin fines de lucro, Entidades } \\
\text { Públicas, empleadoras y empresas } \\
\text { de inserción. }\end{array}$ & $\begin{array}{l}\text { Personas con } \\
\text { discapacidad } \\
\text { o con riesgol } \\
\text { situación de } \\
\text { exclusión social. }\end{array}$ & 21.455 .000 & 21.900 .000 & 22.600 .000 & $\begin{array}{l}\text { SEPE } \\
\text { GV } \\
\text { NC }\end{array}$ \\
\hline S2243000 & 7 & $\begin{array}{l}\text { Inversión para fomento } \\
\text { del empleo para personas } \\
\text { con discapacidad. }\end{array}$ & A. 3 & $\begin{array}{l}\text { Empresas, Centros Especiales de } \\
\text { Empleo e Instituciones sin fines } \\
\text { de lucro y otros empleadores. }\end{array}$ & Desempleados. & 100.000 & 100.000 & 100.000 & SEPE \\
\hline
\end{tabular}

FUENTE: SERVEF (2017). Elaboración propia.

TABla 4. Programa presupuestario: 322.52 Formación y cualificación profesional (Importes anuales, vinculación con objetivos operativos y fuentes de financiación)

\begin{tabular}{|c|c|c|c|c|c|c|c|c|c|}
\hline $\begin{array}{c}\text { Código } \\
\text { Línea }\end{array}$ & Cap. & Descripción & Obj. & Solicitantes & $\begin{array}{l}\text { Colectivo destinatario } \\
\text { Participantes }\end{array}$ & $\begin{array}{c}\text { Importe } \\
\text { proyecto } \\
2017\end{array}$ & $\begin{array}{c}\text { Importe } \\
\text { proyecto } \\
2018\end{array}$ & $\begin{array}{c}\text { Importe } \\
\text { proyecto } \\
2019\end{array}$ & Fin. \\
\hline S7994000 & 4 & $\begin{array}{l}\text { Alumnado asistente a los } \\
\text { talleres deformación e } \\
\text { inserción laboral Orden } \\
56 / 2010 \text { CEHE. }\end{array}$ & B.1. & Personas físicas. & $\begin{array}{l}\text { Colectivos con } \\
\text { difcultades deinserción } \\
\text { social, inmigrantes y } \\
\text { personas con discapacidad. }\end{array}$ & 62.700 & 0 & 0 & GV \\
NC \\
\hline
\end{tabular}

FuENTE: SERVEF (2017). Elaboración propia.

De igual modo, según se indica en la Memoria de actividades del SERVEF de 2018 (SERVEF, 2018), la Subdirección General de Empleo enfatiza los colectivos que afrontan mayores dificultades de inserción laboral, atendiendo, a su vez, a la vertiente local y sectorial del empleo. Las principales actuaciones se han centrado en los siguientes puntos:

- La integración sociolaboral de trabajadores/as con discapacidad en empresas ordinarias (dotación de 1.400.000€).

- Fomento del empleo para personas con discapacidad en CEE y enclaves laborales (dotación inicial de 19.660.000 € y final de 26.129.012,48 €). 
- Fomento del empleo destinado a la creación o mantenimiento de las Unidades de apoyo a la actividad profesional como medida de fomento del empleo para personas con discapacidad en CEE (dotación de 1.200.000 €).

Desde la Subdirección General de Formación Profesional para el empleo se han desarrollado también acciones formativas de diversa índole:

- Colaboración con Fesord (Federación de personas sordas): $25.000 €$.

- Formación Modalidad Colectivos. Beneficiarios: centros/entidades de formación, inscritas o acreditadas, que ofrecen formación adaptada a las necesidades del colectivo entre los certificados de profesionalidad y especialidades incluidas en el Fichero de Especialidades Formativas del SEPE, con 1.241.730,00€.

Ha participado en esta modalidad exclusivamente el alumnado siguiente:

a) Personas en situación o riesgo de exclusión social.

b) Personas con parálisis cerebral, enfermedad mental o discapacidad intelectual con un grado igual o superior al $33 \%$ y personas con discapacidad física o sensorial con un grado igual o superior al $65 \%$.

\section{Conclusiones}

El texto plantea cómo la participación social y la inclusión laboral son ejes fundamentales en el desarrollo de las personas con discapacidad para mejorar su calidad de vida social, profesional y personal y, por lo tanto, su autodeterminación, su bienestar general y su constitución como ciudadanía con plenos derechos.

En este sentido, en respuesta a la primera cuestión de la investigación, tras la revisión de algunos ejemplos de políticas autonómicas relacionadas con la inserción laboral, puede afirmarse que la mejora de la empleabilidad impulsada desde las políticas públicas tiene una incidencia clara en la mejora de su calidad de vida. A nivel macro, favorecen la incorporación a los puestos de trabajo las medidas de fomento de empleo, los programas de empleo con apoyo y de formación, el acceso al empleo público por reserva de plaza, etc. Desde una visión micro, es necesario tener presente un prisma de valor e igualdad para cada persona que es diversa funcionalmente, preservando así su dignidad y evitando la discriminación.

En cuanto a la segunda cuestión del trabajo, estamos en condiciones de poder afirmar que los sectores implicados (público, empresarial y social), destacan la necesidad de la incorporación de la responsabilidad social y, en menor medida, la gestión de la diversidad, como instrumentos para la inclusión de personas con discapacidad.

Asimismo, que su relevancia en la gestión actual de las empresas y los retos que se plantean por la transformación del mercado laboral refuerzan el carácter transversal de ambas cuestiones, muy presentes en los Objetivos de Desarrollo Sostenible 2030.

Por todo ello, la revisión de las políticas responsables de las administraciones permite concluir que, pese a las medidas desarrolladas en su compromiso con la inclusión social de las personas con discapacidad, todavía queda mucho camino por hacer 
como: adaptar las pruebas de acceso a las funcionalidades específicas de este colectivo heterogéneo, regular que las empresas cumplan la normativa de reserva de puestos de trabajo, elevar la cuota de reserva o acumular los porcentajes cuando existan vacantes.

Asimismo, según las funciones de las administraciones como impulsoras de estas políticas a los servicios de empleo, pueden sugerirse diferentes actuaciones pendientes que se exponen de forma esquemática:

Función Reguladora:

- Generar medidas que garanticen la compatibilidad de las prestaciones con los salarios, recompensando a quienes se reincorporan al empleo y cuidan su salud y su productividad, para evitar, al menos parcialmente, la desmotivación a insertarse laboralmente dados los bajos sueldos percibidos.

Función Colaboradora:

- Gestar redes con los centros de formación profesional y las empresas e, igualmente, entre los diferentes organismos competentes en la materia.

Función Facilitadora:

- Estimular la implicación de todos los actores clave en la inserción sociolaboral para la búsqueda de iniciativas innovadoras, entendiendo por tales no solo las empresas, los sindicatos o las entidades formativas, sino también las familias o las propias personas con discapacidad a través de las organizaciones representativas de sus intereses.

- Medidas de fomento de políticas responsables por medio de incentivos, formación de personal directivo, campañas de sensibilización y concienciación a agentes sociales y empresariales en temas como: adaptación razonable de los puestos y lugares de trabajo; eliminación de los temores a las posibles alteraciones que personas singulares y diferentes pudieran introducir en el ambiente laboral; divulgación de buenas prácticas en general y, específicamente, para las pymes y microempresas, no obligadas al cumplimiento de la cuota de reserva, etc.

Función Promotora:

- Desarrollar fórmulas de reconocimiento directo de los esfuerzos y actitudes positivas y comprometidas (p. e., premios), financiar consultoras para asesorar a pymes y fomentar las metodologías para su implantación. Todo ello, siempre teniendo en cuenta que el colectivo al que van dirigidas estas medidas es muy heterogéneo, por lo que es necesario particularizar los perfiles en las actuaciones.

Actuaciones de los propios servicios públicos de empleo:

- Acciones orientadas al asesoramiento, información y divulgación para conseguir que las personas con discapacidad pasen a ser demandantes activos de empleo, incluyendo una adecuada evaluación de las necesidades y de los puntos fuertes de las personas demandantes de trabajo. 
- Formación específica de profesionales y constitución de equipos especializados en los servicios de intermediación laboral.

- Formación Profesional Ocupacional adaptada a diferentes tipos de discapacidad.

- Mecanismos activos de seguimiento y contacto con las empresas tras las contrataciones, buscando mayores niveles de estabilidad y consolidación del empleo.

- Promoción de intervenciones tempranas de (re)incorporación al trabajo.

Sobre esta base, siguiendo a Ibáñez (1997), para trazar nuevas alternativas hay que conjugar dos operaciones: una distinción - una frontera que las genere: un nuevo servicio, una nueva forma de producción o un modelo diferenciado de empresa- y una indicación - una preferencia por uno de los lados de la frontera-; es decir, la elección entre las estrategias de:

a) vía estrecha, meramente instrumental, consistente en presionar a la baja sobre los costes de los factores, en los que la responsabilidad y la inclusión de las personas con discapacidad consisten en una moda pasajera;

b) vía ancha, estratégica, que impulse un proceso activo de aprendizaje y creatividad que desarrolle políticas inclusivas, en la que ambos instrumentos pueden resultar adecuados para la incorporación de la pluralidad en los espacios de trabajo.

Pero realizar el segundo camino solo será posible desde una responsabilidad social estratégica e integradora, que optimice la gestión interna de las empresas y sus relaciones con el entorno, facilitando la participación de los skateholders. Una responsabilidad social que, sin negar el legítimo objetivo de conseguir beneficios sostenibles en el tiempo, aporte procesos operativos que valoren la capacidad de producción de las personas con discapacidad, así como su contribución social y su aportación personal a la empresa. Actuaciones que aseguren la calidad de vida aprovechando el conocimiento del entorno y las sinergias de los actores y recursos locales en aras del bien común.

\section{Referencias bibliográficas}

Aguilar, P. y Yusta, N. (2017). La Inserción laboral de las personas con discapacidad. 3C Empresa: Investigación y Pensamiento Crítico, 6(2), 1-19.

BA. (2012a). Los discapacitados entran en plantilla. Boletín de Actualidad. Barcelona: Barcelona Activa, Ajuntament de Barcelona, 03 de marzo.

BA. (2012b). Discapacidad y búsqueda de empleo. Boletín de Actualidad. Barcelona: Barcelona Activa, Ajuntament de Barcelona, 24 de marzo.

Batt, R. y Valcour, P. (2003). Human Resources Practices as Predictors of Work-Family Outcomes and Employee Turnover. Industrial Relations, 42(2), 189-220.

Canyelles, J. M. (2011). Responsabilidad social de las administraciones públicas. Revista de Contabilidad y Dirección, 13, ACCID, 77-104.

Carneiro, M. (2004). La Responsabilidad Social Corporativa interna: la "nueva frontera" de los Recursos Humanos. Madrid: ESIC.

Castillo, M. y Suso, A. (2012). La Gestión de la diversidad: por una estrategia de gestión de la edad y de la discapacidad en las empresas. Madrid: Fundación ONCE. 
CCE. (2001). Libro Verde. Fomentar un marco europeo para la responsabilidad social de las empresas. Bruselas: Comisión de las Comunidades Europeas, COM (2001) 366 final, 18 de julio.

CD. (2006). Libro Blanco: Informe de la Subcomisión para potenciar y promover la responsabilidad social de las empresas. Boletin Oficial de las Cortes Generales, VIII Legislatura, 4 de agosto. Madrid: Congreso de los Diputados.

CE. (2011). Estrategia renovada de la UE para 2011-2014 sobre la responsabilidad social de las empresas. Comunicación de la Comisión al Parlamento Europeo, al Consejo, al Comité Económico y Social Europeo y al Comité de las Regiones. Bruselas: Comisión Europea, COM (2011) 681 final, 25 de octubre.

Chaves, R. y Monzón, J. L. (2018). La economía social ante los paradigmas económicos emergentes: innovación social, economía colaborativa, economía circular, responsabilidad social empresarial, economía del bien común, empresa social y economía solidaria. CIRIEC-España, Revista de Economía Pública, Social y Cooperativa, 93, 5-50. http://dx.doi. org/10.7203/CIRIEC-E.93.12901

Claes, C., van Hove, G., Vandevelde, S., van Loon, J. y Schalock, R. (2012). The influence of support strategies, environmental factors, and client characteristics on quality of liferelated personal outcomes. Research in Developmental Disabilities, 33, 1, 96-103. http:// dx.doi.org/10.1016/j.ridd.2011.08.024

Cox, T. H. y Blake, S. (1991). Managing cultural diversity: implications for organizational competitiveness. Academy of Management Perspectives, 5, 3, NY. http://dx.doi. org/10.5465/ame.1991.4274465

Cummins, R. A. (1997). Comprehensive quality of life scale-intellectual/cognitive disability. Melbourne: Deakin University.

Dalmau, N., Sala, I. y Llinares, M. (2015). Análisis de la formación universitaria recibida por los titulados universitarios españoles con discapacidad auditiva en la adquisición de las competencias profesionales. Siglo Cero, 46(3), 27-46. http://dx.doi.org/10.14201/scero20154632746

Díaz, O. C. (2005). Equidad, inclusión y discapacidad. En Memorias II Foro Distrital de Discapacidad: "En nuestra Bogotá sin indiferencia reconozcamos la diferencia", octubre de 2004, Bogotá, 18-24.

Drucker, P. F. (1999). La sociedad postcapitalista. Buenos Aires: Ed. Sudamericana.

EERSE. (2014). Estrategia 2014-2020 para empresas, administraciones públicas y el resto de organizaciones para avanzar hacia una sociedad y una economía más competitiva, productiva, sostenible e integradora. Madrid: Ministerio de Empleo y Seguridad Social, julio.

Felce, D. y Perry, J. (1995). Quality of life: its definition and measurement. Research in Developmental Disabilities, 16(1), 51-74.

Forcada, C. I. (2015). La inserción de personas con discapacidad en el mercado laboral privado. Invenio, 18(35), 85-104.

Fox, T., WARD, H. y HowARD, B. (2002). Public sector roles in strengthening Corporate Social Responsibility: a baseline study. Washington: The World Bank.

Fund. Adecco. (2015). IV Informe Adecco sobre Absentismo. Madrid: Fundación Adecco, IESE, Garrigues, Fremap, Universidad Carlos III de Madrid, AMAT.

Fund. Adecco. (2018). Jóvenes con discapacidad, motor de futuro. Madrid: Fundación Adecco.

Fund. Alternativas. (2009). Informe La Responsabilidad Social Corporativa en España 2009. Madrid: Fundación Alternativas.

Fund. Cepaim. (2016). Responsabilidad Social, Ética Empresarial y Gestión de la Diversidad. Cuaderno para la formación. Madrid: Fundación Cepaim. 
GonZÁlez, J. (2019). Intensidad de apoyos, salud mental, empleo y su relación con resultados de calidad de vida. Siglo Cero, 50(2), 73-88. http://dx.doi.org/10.14201/scero20195027388

González, C. y Naranjo, G. (2015). La responsabilidad institucional pública: un análisis para el mejoramiento de la gestión y la gerencia en la administración pública. Revista CIFE: Lecturas de Economía Social, 17(27), 245-292.

Gracia, E., Vidal, N. y Martínez, V. (2017). La calidad de servicio desde el punto de vista de las personas con discapacidad intelectual: relaciones con su satisfacción y bienestar. Siglo Cero, 48(3), 41-53. http://dx.doi.org/10.14201/scero20174834153

IвÁÑ̃E, J. (1997). A contracorriente. Madrid: Fundamentos.

IEGD. (2019). Gestión de la diversidad en empresas e instituciones. Castellbisbal, Barcelona: Instituto Europeo para la Gestión de la Diversidad.

Izuzquiza, D. y Rodríguez, P. (2016). Evaluación de la metodología Empleo con Apoyo (ECA) en el Programa Promentor (UAM-PRODIS). El ajuste competencial. Siglo Cero, 47(1), 37-54. http://dx.doi.org/10.14201/scero201613754

Lukas, J., Etxeberria, J., Galarreta, X. y Lizasoain, L. (2016). Construcción y validación de la escala de satisfacción laboral (ESL) en centros especiales de empleo. Siglo Cero, 47(4), 57-72. http://dx.doi.org/10.14201/scero20164745772

Melle, M. (2014). La responsabilidad social en el sector público. Revista de Responsabilidad Social de la Empresa, 18(III), 17-43.

Mercado, E., Roldán, E. y Rivera, J. M. (2016). El perfil de los preparadores laborales en el empleo con apoyo. Siglo Cero, 47(3), 69-88. http://dx.doi.org/10.14201/scero20164736988

Morán, M., Gómez, L. y Alcedo, M. (2019). Inclusión social y autodeterminación: los retos en la calidad de vida de los jóvenes con autismo y discapacidad intelectual. Siglo Cero, 50(3), 29-46. http://dx.doi.org/10.14201/scero20195032946

Morrós, J. y VidAL, I. (2005). Responsabilidad Social Corporativa. Madrid: Fund. Confemetal Ed.

OIT. (2014). Informe Lograr la igualdad de oportunidades en el empleo para las personas con discapacidades a través de la legislación. Directrices. Ginebra (Suiza): Organización Internacional del Trabajo, 25 de noviembre.

ORSC. (2011). Posicionamiento del Observatorio de RSC sobre Políticas Públicas y RSC, 2011. Madrid: Observatorio de Responsabilidad Social Corporativa, septiembre.

Palacios, A. y Romañach, J. (2006). El modelo de la diversidad: la Bioética y los Derechos Humanos como herramientas para alcanzar la plena dignidad en la diversidad funcional. A Coruña: Diversitas-AlES.

Patston, P. (2007). Constructive functional disability: a new paradigm beyond disability and impairment. Disability and Rebabilitation, 29(20-21), 1625-1633.

Porter, M. E. y Kramer, M. R. (2006). Strategy and society: the link between Competitive Advantage and Corporate Social Responsibility. Harvard Business Review, diciembre, 78-92.

CE. (2014). Posición de los servicios de la Comisión sobre el desarrollo del Acuerdo de Asociación y de programas en España en el período 2014-2020 (Exclusivamente para uso interno, no destinado a publicación). Bruselas: Comisión Europea [en línea] <https://www.dgfc.sepg.hacienda.gob.es/sitios/dgfc/esES/ipr/fcp1420/p/di/Documents/PP\%20España\%20(ES).pdf>.

Raufflet, E., Lozano, J., Barrera, E. y García, C. (2012). Responsabilidad social empresarial. México: Pearson.

Roosen, K. (2009). From tragedy, to “crip", to human: the need for multiple understanding of disability in psychotherapy. Critical Disability Discourse, 1, 1-25.

Rubio, J. (2006). La exclusión sociolaboral de colectivos con dificultades en su acceso al mercado. Nómadas. Critical Journal of Social and Juridical Sciences, 14(2), 1-8. 
Ruiz Olabuénaga, J. I. e Ispizua, M. A. (1989). La decodificación de la vida cotidiana: métodos de la investigación cualitativa. Bilbao: Universidad de Deusto.

Ruiz Otero, E., Gago, M. a L., García, C. y López, S. (2012). Recursos humanos y responsabilidad social corporativa. Madrid: McGraw-Hill.

Sánchez, A., Hernández, M. y Fernández, M. (2016). Estrategias metodológicas para el estudio de la inserción sociolaboral de las personas con discapacidad. Revista Española de Discapacidad (REDIS), 4(1), 97-117.

Schalock, R. L. y Verdugo, M. Á. (2007). El concepto de Calidad de vida en los servicios y apoyos para personas con discapacidad intelectual. Siglo Cero, 38(4), 21-36.

SEN, A. (2002). Rationality and Freedom. Cambridge (Mass.): Belknap.

SERVEF. (2010). Catálogo de servicios de 2010 del SERVEF. Valencia: SERVEF - Conselleria de Economía Sostenible, Sectores Productivos, Comercio y Trabajo.

SERVEF. (2016). Plan Estratégico del SERVEF 2016-2022. Valencia: SERVEF - Conselleria de Economía Sostenible, Sectores Productivos, Comercio y Trabajo.

SERVEF. (2017). Plan Estratégico de subvenciones 2017-2019. Valencia: SERVEF - Conselleria de Economía Sostenible, Sectores Productivos, Comercio y Trabajo.

SERVEF. (2018). Memoria de actividades del Servef. Valencia: SERVEF - Conselleria de Economía Sostenible, Sectores Productivos, Comercio y Trabajo.

TAMARIT, J. (2015). La transformación de los servicios hacia la calidad de vida. Una iniciativa de innovación social de FEAPS. Siglo Cero, 46(3), 47-71. http://dx.doi.org/10.14201/scero20154634771

The Conference Board. (2006). The business case for diversity: good practices in the workplace. Nueva York: The Conference Board, February. <https://www.conferenceboard. $\mathrm{org} /$ publications/publicationdetail.cfm?publicationid $=1099>$.

Thomas, D. A. y Ely, R. J. (1996). Making differences matter. Harvard Business Review.

Valles, M. S. (1997). Técnicas cualitativas de investigación social. Reflexión metodológica y práctica profesional. Madrid: Síntesis.

Vived, E. y Delgado, L. (2016). Un estudio sobre la eficacia en la estructuración de los apoyos en formación profesional para jóvenes con discapacidad intelectual. Siglo Cero, 47(2), 99114. http://dx.doi.org/10.14201/scero201647299114

Whoqol Group. (1995). The World Health Organization Quality of Life Assessment (WHOQOL): position paper from the World Health Organization. Social Science and Medicine, 41(10), 1403-1409. http://dx.doi.org/10.1016/0277-9536(95)00112-.k 